\title{
The Current Issue of the Digital Game Concept Art Education for Chinese Undergraduates
}

\author{
Xiang Chaochu
}

\begin{abstract}
In recent years, the development of the game industry in the global market is very strong. The market has spawned the major art colleges to promote the course abou t the game, especially the digital game concept art. But now there is still some disconnects between the corresponding talent training ideas and the actual market demand. Relevant theoretical research results also need to be further deepened. This article will take the job characteristics of the original game as the starting point, analyze with the actual situation of the current teaching practice, and research the crux of the current talent training model. And suggest making new innovative measures to be the corresponding solutions, such as develop new evaluation criteria, introduce project-based teaching, and linkage of different courses. I hope to provide some useful thoughts for cultivation of talents and related teaching reform in the game art industry.
\end{abstract}

Index Terms-Digital game concept art, game industry, traditional painting, computer education.

\section{INTRODUCTION}

In the past 10 years, the development of the game industry in the world has sprung up. In 2009, the total value of game games in the United States was only 19 billion US dollars. In 2012, the output value of a single game was very amazing: Activision Blizzard is 13 billion US dollars, Zynga is 9 billion US dollars, EA is 8 billion US dollars, etc. The total output value about the stand-alone game in major companies reached $\$ 37.63$ billion. And the development of the domestic market is similar. In 2015, the revenue of mobile games in China increased by nearly 10 times compared with 2012 [1] in 2016, the number of domestic game users reached 489 million, and the actual sales of the Chinese game market reached 78.75 billion yuan, which has an increase of $30.1 \%$ over the same period of 2015 [2].

The fast-growing in game industry has also triggered a dramatic chain reaction in the domestic education community. Many art colleges have quickly launched corresponding courses in the direction of game art. However, such a sharp change in teaching naturally has some practical problems. The contradiction in education at the digital game concept art is particularly prominent, resulting in an unsatisfactory employment situation for graduates in every year. To solve the current teaching problems, we need to define the working characteristics and corresponding characteristics of the digital game concept art at first, so that we can make a

Manuscript received January 13, 2019; revised April 13, 2019.

The author is with Chengdu University, China (e-mail: 254494839@qq.com). reasonable teaching orientation for its characteristics.

\section{DEFinition And Function of Digital Game ConcePt ART}

According to the CMP Game Group survey, which provides professional information inquiry for the game industry, there are about 39,700 practitioners and sales professionals engaged in the game development in the United States. And personnel engaged in the development of around $65 \%$ are art staff. The most important job is the original artist [3] So what exactly is the digital game concept art?

The digital game concept art is a design draft depicting specific game elements, including characters, scenes, props, etc., which is a very important and independent job to help industrial production. [4] The digital game concept art can be divided into two categories, and its emphasis is also different: the first category is conceptual painting, its function is propaganda, requiring the screen to have a strong visual appeal: for example, the character shape is exquisite, dynamic, light and shadow rich and true. The second category is design painting, which is responsible for the rigorous design of all the characters in the game, emphasizing the logic and rigor of the design, as well as the expression of specification.

Conceptual painting works in the early stage of game production, the original artist draws the corresponding characters and scenes according to the documents. The drawing of this stage requires repeated scrutiny in terms of comprehensive modeling, color, artistic atmosphere, etc., and finally determines the overall art style of this game. Conceptual painting has relatively strong creativity, and it also has very strict requirements for uniformity. It is necessary to realize the unified coordination between roles, roles and scenarios. This is why the market is more demanding to the conceptual painting designer. The design painting is mainly based on the conceptual painting, and it is rigorous and logically refined, and it designs the specific details, structure, color and material relationship, etc. The design painting provides guidance standards and reference opinions to the medium-term team such as the mold and mapping department. Design painting is a very important part of game production. Its normative and logical nature is especially important. The expression of each form must be accurate so that the follow-up work can proceed smoothly.

Therefore, the digital game concept art is to pre-plan and locate the art image and art style of the whole game, and practically guide the subsequent work such as modeling, mapping, animation, rendering. The digital game concept art not only largely determines the production mode, production 
difficulty, and production cost of the game art in the middle and later stage, but also plays a key role in the final pictures of the game. And picture of the game is tied with the game story, game interaction for three important criteria. Therefore, the digital game concept art is the most valuable part of the design in the whole game art work, and its status is also crucial.

For example, the "Call of Duty" series, which is the flagship single-player game of the famous Activision game company in the United States, has achieved great success in the "Call of Duty - Modern Warfare" series, which has been designed with good conceptual painting and design painting. The "Call of Duty - Modern Warfare" series created an amazing sales volume, especially the third season was the most, reaching an unprecedented 30.33 million copies. But the" Call of Duty - Black Ops" series ushered in a buzz and return frenzy by a rough work. Until two years later, the "Call of Duty - Black Ops II" was finally accepted by the market though greatly enhanced art design. The difference in market feedback fully demonstrates the important role of excellent digital game concept art in the same post-production team, the same game engine, and the same game product launched by a company. There are similar cases in mobile platform games. The "N.O.V.A. 3" series and the "Infinity Blade" series of games have far surpassed similar game products in visual effects and achieved great commercial success, which with a reasonable engine and good digital game concept art

These cases have proved the great effectiveness of excellent digital game concept art, and also proved the primacy of the original painting design. Such an important original painting design work, what problems exist in the current university related teaching?

\section{CURRENT EduCATION ISSUE}

Nowadays the basic teaching framework of most domestic art academy is based on the Western modern art education system which was popular in many years ago. The traditional pure art painting is still a leading role in ideology. The art concept of traditional painting is to reflect the theme of authenticity through the picture, and its core concept emphasizes in unique painting expression reveals the truth of life, history and culture, reflecting the style and characteristics of the time. The traditional painting emphasizes a kind of cultivation about artistic personality. At present, many colleges and universities for the teaching thinking of digital game concept art in a very great degree is still affected by the above concept. They represent a typical traditional education thought: their teaching orientation emphasizes a long-term basic training and a relatively abstract understanding of thinking model. They put students as the object of teaching, supplying systematic education for them and emphasizing the role of the educators. [5] When students have mastered basic skills on body structure painting, light and shadow relationship and color relationship, they focus on the cultivation of abstract deformation and artistic expression. Many teachers used to instilling students with the concepts of "feeling", "inspiration" and "multi-painting". This kind of teaching orientation has a serious deviation from the market demand of the digital game concept art. Naturally, it has also produced many problems. The most outstanding is mainly the following points:

\section{A. Lack of Standard and Uniform Training}

Although the digital game concept art has many similar and overlap with traditional paintings in terms of drawing techniques and artistic expression, its core concept has always been a design behavior that guides production of the game industry in the middle and late stage. However, current teaching orientation about emphasizing individuality makes the corresponding courses seriously lack of normative links in design, which leads many students to seriously lack the corresponding training in the four years of study. Even in the game company's recruitment test, students unreasonably explained their wrong body structure painting are "artistic" and "postmodern". How can we produce a precise original draft according to a painting concept which is not strictly in accordance with industry norms? How can an original draft which is lack of normative to guide the specific production work of the middle and late departments? How can we create a game product that can withstand the test of the market? Therefore, the lack of normativeness is the most serious problem in the current digital game concept art education.

Every art course training is a process from basic practice to creative innovation. The innovation of the digital game concept art is not only needs the standard and innovation to each individual prop role, but also emphasizes the unified integration of design ideas into a series of props, characters and scenes. And in this series of highly unified design can clearly show the characteristics of each object. This is a relatively neglected part of current domestic digital game concept art education. Current education emphasizes the evaluation of individual works, and has not involved too much on the linkage evaluation of multiple works. Therefore, according to the feedback from relevant companies, a part of students who have a good painting skills are able to complete a single original painting that meets the test standards every year. Once they are faced to the design of unified series work, they will be embarrassed. Even if they are hired, they'll take a long time to adapt to the work. In the actual design for digital game concept art, the intensity of the work about using uniformity is very hard. Therefore, in order to cultivate excellent talents for digital game concept art, we must strengthen the specific training for uniformity.

\section{B. Ignore the Important of Logic and Timeliness}

Relatively speaking, pure art painting is not too timeliness requirements. Because each piece of painting is equivalent to a finished product, and because of the characteristics of its pigments, many paintings can be drawn over a large time span, even allowing in the process of drawing, the painter repeatedly arbitrarily modifies according to his own artistic sentiment. This kind of painting idea has also been brought into the education of the digital game concept art, especially the teachers who have been engaged in traditional painting for many years. They usually think the digital game concept art as "the painting behavior done by computer as the medium", which is reflected in excessive pursuit of the picture effect and detail. Or pursue painting training that does not conform to the current game art style or theme. 
The working characteristics of the digital game concept art determine the drawing way in assembly line nature. Each step is in a strict order: for example, how to separate the line draft? How to flatten the color block and draw the shadow distribution? What is the difference in brightness between colors? And how to soften the edges and add complementary colors, etc. Steps of the whole painting are extremely logical, and there are corresponding strict restrictions. This is a work method that summarizes from a large number of long-term practical work and maximizes efficiency under the premise of ensuring the quality of work. The core of the digital game concept art is the service. If the digital game concept art is not completed on time, the following woks such as modeling, mapping, code, and special effects will be stopped, and the entire construction period will be delayed. Now the competition in the game market is extremely fierce, which will bring serious consequence as a result. Because the market and production characteristics determine the strong timeliness of the digital game concept art, in order to achieve the maximum timeliness, it is necessary to carry out logical operations at the drawing. How to teach students this kind of virtuous circle of thinking, this is what we need to think about the current teaching.

\section{Negligence in Teamwork}

Teamwork is also a big difference between digital game concept art and traditional painting. The "National Oil Edition" in pure art can achieve personal creation and independence finish to a certain extent. The painter can complete related creations separately following his own creative concept as a freelancer. Therefore, under this kind of thinking, the corresponding teaching will not emphasize the training for students' teamwork ability, and naturally lack the cultivation of students' coordination and communication skills.

But game production is a team behavior, and the requirements for the concept designer's communication and collaboration skills in this team are far greater than other people. First of all, the concept designer is the main landscaping, they should have good communication with the copywriter, understand the design intent of the game plan, transfer the concept image to the other concept designer and guide them to complete the corresponding painting work, and then build with the later works. And then, the concept designer communicate interactively with modeling and texturing workers, making an effective communication about the game artistic conception and specific design work. To a large extent, the concept designer is at the core of the entire game team. On the one hand, concept designer's work is most important in the design works of the whole game, and on the other hand, concept designer is the key that makes the whole team work well. It is hard to imagine a graduate who has strong painting and design skills but is not good at effective communication and collaboration. How does he can be qualified for the work of the concept designer?

\section{THE CORRESPONDING SOLUTION}

The core problem of the problems exposed in the teaching of digital game concept art is that the development of the game industry in recent years is much faster than the adjustment of professional teaching. As a result, some of the teachers have not completely transformed from the traditional art thinking of pure art to the new educational thinking which should be close the game industry's production characteristics, the production characteristics of digital game concept art, and be highly linked to the game project.

Anyway, we still believe that the development of the game industry in future is still able to maintain a long-term prosperity. This is because the accelerating rhythm of life and the advent of the era of reading pictures, the game with the three characteristics of sight, hearing and interaction has become the most suitable way of entertainment for the contemporary people's life. And the game is far ahead of any other digital entertainment which owns a great advantage of extremely interactive. Furthermore, thanks to the rapid development of computer hardware and game engine technology in the information age, the artistic expression and gameplay of the game itself will continue to achieve a sustained leap. Meanwhile, the rise of mobile platforms has brought more vitality to the development of the game industry. Mobile games are also the strongest in the game industry in all areas: since the first generation of iPhones in China, the customer number of Chinese mobile game industry has increased 51 times in 8 years, and its market size is even more surged from 240 million yuan in 2008 to 37.48 billion yuan. [6] Compared with the game's performance and performance platform's rapid transformation, the link of concept design which is in the early period of the game design, its corresponding work mode and even the personnel ratio have been relatively stable in the past ten years of development. Therefore, we have reason to believe that in the future market, the concept design will be a very important and relatively stable employment channel for art graduates. So regarding the teaching of the digital game concept art, we need to explore a talent training model that conforms to the rules of the market.

In view of the many problems existing in the current teaching links, we should carry out the innovations in teaching thinking at first, strictly following the development trend of the industry and the specific norms of the industry, and setting up a scientific and rational curriculum system. The curriculum system should firstly adjust the teaching content and evaluation criteria of the basic foundation courses such as modeling foundation, color thinking and design thinking to the work requirements of the digital game concept art, and cultivate a good professional foundation for the freshmen. In addition to the training of the basic color ability in the styling training stage, it is necessary to carry out intensive training for game props and characters. It is suggested that the painting objects in the traditional sketching, color sketching and other courses can be extended to the surrounding products such as models and hand-made games, so as to establish the students' early understanding of the concept design and color matching of the game. Moreover, in the professional basic training stage, students should be taught how to collect and type the materials, and do the basic work for the later professional skills courses.

After completing the professional foundation training, the 
actual game art project should be introduced into the classroom by the project-based teaching method, and it should be used as the teaching line of the follow-up course. Let students train in the form of a team and strictly follow the industry standards of the digital game concept art. In this stage, emphasis will be placed on the corresponding training of normative, uniform and time-sensitive, and reasonable evaluation criteria will be established for it as an important part of the final grade of the course. In this way, students can understand the essence of the digital game concept art, master the workflow and industry norms, obtain good design thinking and drawing ability, and ensure that the design ability of the digital game concept art is improved.

Furthermore, it is recommended to link the concept design of the game with the follow-up course. The training assignments of the concept design can be provided disorderly to other students as a training blueprint for the middle and late course links of the second and third-dimensional courses, such as the middle frame, modeling, and texturing. And in the follow-up course teaching, the concept designer is responsible for communication and coordination with the producers of the middle and late stages, further strengthening the teamwork ability and comprehensive quality of the students.

Teaching according to such a curriculum system can ensure that students get the professional qualities that meet the market demand to the greatest extent, so that students can truly apply what they have learned, and deliver more excellent and more vital talents to the field of digital game concept art in the game industry of China.

\section{REFERENCES}

[1] GPC, CHG, IDC, 2016 China Game Industry Report, 2016, p. 15

[2] B. Lu et al., Annual Report on Development of Chinese Animation Industry 2016, Social Sciences Press, Oct. 2016, p. 90

[3] D. Lin, Introduction to the Development of the World Animation Industry, Shanghai Jiao Tong University Press, May 5008, p. 44.

[4] B. Y. Shen et al., Game Concept Art Design, Tsinghua University Press, Aug. 2011, p. 31.

[5] Z. Y. Weng, Introduction to Art Education, Apr 2009, p. 175

[6] GPC, CHG, IDC, 2016 China Game Industry Report, 2016, p. 17

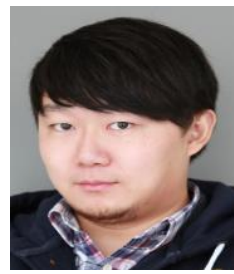

Xiang Chaochu was born in Chengdu city, Sichuan, China on 8th December, 1984. Mr. Xiang graduated from Chengdu University with a bachelor's degree in July 2007. Then he graduated from the Academy of Fine Arts of Sichuan Conservatory of Music with master's degree in July 2012.

In September 2012, he taught at the Academy of Fine Arts of Chengdu University as a teaching assistant. In 2015, he was promoted to lecturer title. In 2017, he taught at the Chengdu University Film and Animation School. The main research background is Japanese animation, animation sociology, animation industry and so on. His main academic research results: "The Success of Japanese Anime from the Perspective of Gundam", Chinese Core Journal Criterion of $P K U$, Movie Literature, vol. 511, p.73. "The Sword and Traditional Culture in Japanese Anime", Chinese Core Journal Criterion of PKU, Movie Literature, vol. 612, p.73. "The Function of TV Animation in Japanese Industry”, CSSCI, Contemporary Cinema, vol. 12, p. 161. 